\section{Mepolizumab als Zusatztherapeutikum bei eosinophiler COPD}

Pavord ID et al. Mepolizumab for Eosinophilic Chronic Obstructive Pulmonary Disease. N Engl Med 2017; 377: 1613 - 1629

Mepolizumab ist ein humanisierter monoklonaler Antikörper und kann bei Patienten mit Asthma die Rate akuter Exazerbationen senken. Ob Mepolizumab auch als Zusatztherapeutikum bei eosinophiler COPD wirksam und sicher ist, haben Pavord und Kolleginnen/Kollegen jetzt in zwei klinischen Phase-III-Studien untersucht.

Mepolizumab bindet an das Zytokin Interleukin-5 (IL-5), das bei der Entwicklung, Aktivierung und Differenzierung von Eosinophilen beteiligt ist, und wird aufgrund seiner entzündungshemmenden Eigenschaft bei schwerem Asthma eingesetzt. Da Patientinnen und Patienten mit chronisch obstruktiver Lungenerkrankung (COPD) ebenfalls eine Eosinophilie aufweisen können, haben Pavord und sein Team das Biologikum im Rahmen der beiden randomisierten kontrollierten und doppelblinden Phase-IIIStudien METREX und METREO an diesem Patientenkollektiv getestet. Beide Studien wurden von der Pharmaindustrie finanziell gefördert.

\section{Methoden}

Teilnehmerinnen und Teilnehmer beider Studien waren mindestens 40 Jahre alt, litten an einer gesicherten COPD mit $\mathrm{FEV}_{1}$ zwischen 20 und $80 \%$ vom Referenzwert und hatten mindestens eine schwere Exazerbation in den vergangenen 12 Monaten. Eine weitere Voraussetzung war die spätestens drei Monate vor Rekrutierung begonnene Dreifach-Therapie der COPD nach aktuellen Leitlinien, Nichtraucher und Asthma-Patienten wurden ausgeschlossen. Randomisierungskriterium der METREX-Studie war die Eosinophilenzahl, die Patientinnen und Patienten wurden einer von zwei Gruppen zugeteilt:
- Interventionsgruppe (IG): Eosinophilenzahl $\geq 150 / \mathrm{mm}^{3}$ bei Rekrutierung oder mindestens einmal $\geq 300 / \mathrm{mm}^{3}$ in den letzten 12 Monaten.

- Kontrollgruppe (KG): Eosinophilenzahl $\leq 150 / \mathrm{mm}^{3}$ bei Rekrutierung und kein Nachweis eines Wertes $\geq 300 /$ $\mathrm{mm}^{3}$ in den letzten 12 Monaten.

Teilnehmerinnen/Teilnehmer der IG erhielten über 52 Wochen alle vier Wochen 100 mg Mepolizumab subkutan (s.c.), in der KG kam ein Placebo zum Einsatz. Die Dreifach-Therapie der COPD wurde fortgeführt.

In der METREO-Studie zählte der eosinophile Phänotyp für alle Patientinnen/Patienten zu den Einschlusskriterien. Hier erfolgte die Randomisierung 1:1:1 in zwei Interventions- und eine Kontrollgruppe:

- IG1: 100 mg Mepolizumab s.c.

- IG2: 300 mg Mepolizumab s.c.

- KG: Placebo s.c.

Die Injektionen wurden ebenfalls alle vier Wochen über insgesamt 52 Wochen durchgeführt. Es folgte in beiden Studien eine Nachbeobachtungsphase von acht Wochen. Primärer Endpunkt war jeweils die jährliche Rate moderater und schwerer Exazerbationen, zu den sekundären Endpunkten zählten die Zeit bis zur ersten Exazerbation sowie Veränderungen im krankheitsspezifischen Fragebogen (St. George's Respiratory Questionnaire total score SGRQ, COPD Assessment Test (AT) am Ende der Studie im Vergleich zum Ausgangswert. Endpunkte in Bezug auf die Sicherheit waren Art und Häufigkeit von unerwünschten Arzneimittelwirkungen.

\section{Ergebnisse}

In METREX konnten 417 Datensätze der IG und 419 der KG in die finale Auswertung eingeschlossen werden. Bei METREO waren es 223 (IG1), 225 (IG2) und 226 (KG). Teilnehmerinnen und Teilnehmer der IG in METREX hatten durchschnittlich weniger moderate oder schwere Exazerbationen als jene aus der KG $(1,4$ vs. 1,71$)$. In METREO gab es in Hinblick auf den primären Endpunkt keine statistisch signifikanten Gruppenun- terschiede. Patientinnen/Patienten der IG in METREX entwickelten Exazerbationen später als jene der KG, für die Fragebogen konnten in beiden Studien keine bedeutsamen Unterschiede festgestellt werden. Art und Häufigkeit von unerwünschten Arzneimittelwirkungen in den Interventionsgruppen waren sowohl in METREX als auch in METREO vergleichbar mit den entsprechenden Angaben der Kontrollgruppen.

\section{FAZIT}

Der humanisierte monoklonale Antikörper Mepolizumab ist bei Patientinnen/Patienten mit eosinophilem Phänotyp einer COPD sicher und reduziert als Zusatztherapeutikum die jährliche Exazerbationsrate. Die Autorinnen/Autoren sehen in der Eosinophilenzahl einen vielversprechenden Biomarker für spezifische Behandlungsstrategien.

Dipl.-Psych. Annika Simon, Hannover 